\title{
MedEd Symposium 2015: Die Ideen sprudelten
}

\section{Werner Bauer}

Dr. med., Präsident des Schweizerischen Instituts für ärztliche Weiter- und Fortbildung SIWF

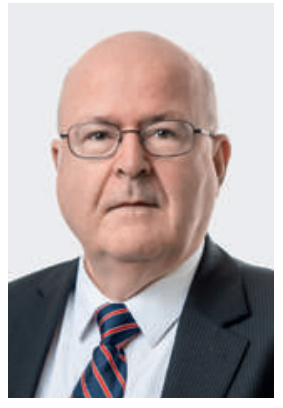

In dieser Ausgabe der Ärztezeitung berichtet der Chefredaktor, Dr. Bruno Kesseli, über das zweite MedEd Symposium, welches das Schweizerische Institut für ärztliche Weiter- und Fortbildung organisiert hat. An der Veranstaltung ging es darum, Überlegungen zur Zukunft der ärztlichen Bildung in Zeiten des raschen Strukturwandels an den Spitälern und im Gesundheitswesen auszutauschen.

Beeindruckend war der Konsens, dass die Weiterbildung zu den Kernaufgaben der Spitäler gehört, auch wenn hie und da aus einem Direktionsbüro verkündet wird, ein Spitalbetrieb liesse sich ohne lernende Assistenzärzte effizienter führen. Der Direktor des Kantonsspitals Winterthur formulierte es so: «Wir wollen ausbilden müssen. Der Nachwuchs fällt nicht vom Himmel.» Was Sir William Osler schon vor über hundert Jahren sagte ("The work of an institution in which there is no teaching is rarely first class.»), bestätigte er: Weiterbildung steigert das Know-how und die Qualität eines Spitals.

\section{Weiterbildung steigert das Know-how und die Qualität eines Spitals.}

Ein Phänomen der Tagung waren die erstaunlich vielen, zum Teil provokativen Thesen, die präsentiert wurden. Sie dokumentieren, dass die Herausforderungen für die ärztliche Bildung zu mannigfachen Überlegungen Anstoss geben. Hier - frei zitiert - eine Auswahl aus dem Bouquet von Vorschlägen, Analysen und Ideen:

- Ärztliche Weiterbildung und Betriebseffizienz müssen sich gegenseitig beachten und respektieren.

- Die Weiterbildung darf Strukturanpassungen nicht verhindern - Strukturanpassungen dürfen die Weiterbildung nicht behindern.

- Die weiterbildenden Institutionen müssen in die Gestaltung der Weiterbildungsprogramme einbezogen werden.

- Wir müssen Wege finden, dem "generalism» in allen Fachgebieten seine Bedeutung zu bewahren oder erst wieder zu vermitteln.

- Von der Weiterbildungsstätte zum Weiterbildungsnetzwerk: Den Netzwerken gehört die Zukunft.
- Netzwerke benötigen eine auf Dauer angelegte Verbindlichkeit, zu vermeiden ist die Hegemonie eines einzelnen Netzwerkpartners.

- Für die Weiterbildung müssen Mittel gesprochen werden, die nicht in einem Gesamtbudget zur Senkung der Fallkosten versickern.

- Mehr Zeit am Bett! Weniger administrative, nichtärztliche Aufgaben! Neue entlastende Berufsbilder sind zu fördern.

Wie wäre es mit Ablösegeldern für top-ausgebildete Spezialisten?

- Fachgesellschaften und Branchenverbände sichern primär die Interessen ihrer Mitglieder: Es braucht deshalb ein starkes, unabhängiges SIWF.

- Wie wäre es mit Ablösegeldern für top-ausgebildete Spezialisten analog den Transfersummen im Sport?

- Maximale Jahresarbeitszeiten, flexiblere Regelung der täglichen und wöchentlichen Arbeitszeiten!

- Die Lernziele von Studium und Weiterbildung sind zu überprüfen. Nach einem verkürzten und verwesentlichten Grundstudium könnten Weiterbildungsmodule schon ins Masterstudium integriert werden.

Auch wenn nicht jede dieser Feststellungen und Anregungen allgemeine Akzeptanz finden wird oder eine Chance hat, realisiert zu werden, verdienen es alle, dass über sie nachgedacht wird. Das Spannungsfeld zwischen den Anforderungen einer überzeugenden Weiterbildung und den Konsequenzen betriebswirtschaftlich orientierter Massnahmen wird nur dann nicht zu gravierenden Konflikten führen, wenn alle Beteiligten frühzeitig die geplanten Änderungen aufeinander abstimmen. Das mag in manchen Fällen leichter gesagt als getan sein, ist aber unumgänglich. Die Weiterbildungsordnung und die Weiterbildungsprogramme sind durchaus flexibel und können wenn nötig - und wenn mit Rücksicht auf die Qualität vertretbar - angepasst werden. Das MedEd Symposium bot eine optimale Möglichkeit, erste Schritte auf neuen Wegen in der ärztlichen Bildung zu skizzieren. 